\title{
Tripled fixed point theorems in partially ordered spaces using a control function
}

\author{
SAVITRI and NAWNEET HOODA \\ Department of Mathematics, DCR University of Science \& Technology, Murthal, India
}

Keywords: Partially ordered metric space, tripled fixed point, control function.

Abstract: In this paper, We prove some new tripled fixed point theorems using a control function which is also referred to as altering distance function.

\section{Introduction}

Fixed point thoerem is a very active field of research at present. Hundreds of reserachers have contributed in this field since 1922 with Banach's fixed point theorem as a milestone. Generalization of this theorem has been a heavily investigated branch of research. Existence of fixed points in ordered metric spaces was investigated in 2004 by Ran and Reurings [19]. Later, so many results were proved on existence and uniquenss of fixed point in partially ordred metric spaces, see e.g. $[2,3,4,8,9,10,11,14,16,18,19,22]$.

In 2011, Berinde and Bocut [2] reported with the notion of tripled fixed point. Later various results on tripled fixed point have been obtained, see e.g. $[1,12,20]$.

The purpose of this paper is to establish some tripled fixed point results in partially ordered complete metric spaces using a control function which is referred to as altering distance function. This control functoin has been heavily used in metric fixed point theory. Some recent references are Choudhury [5], Sastry and Babu [21], Mihet [15], Naidu [17], Choudhury and Das [6] and Dutta and Choudhury [7].

To begin, we first recall the definitions and notations that will be needed in the sequal.

Definition 1.1 [12]. The triple $(X, d, \leq)$ is called partially ordered metric space if $(X, \leq)$ is a partially ordered set and $(X, d)$ is a metric space.

Definition 1.2 [2]. Let $X$ be a non-empty set and $F: X^{3} \rightarrow X$ be a map. An element $(x, y, z) \in X^{3}$ is called a tripled fixed point of $F$ if $F(x, y, z)=x, F(y, x, y)=y, F(z, y, x)=z$.

Definition 1.3 [13]. A function $\psi:[0, \infty) \rightarrow[0, \infty)$ is called an altering distance function if the following properties are satisfied:

(i) $\quad \psi$ is monotone increasing and continuous,

(ii) $\quad \psi(t)=0$ iff $t=0$.

\section{Notation.}

- Througout the paper, we assume that $X \neq \phi$ and $X^{n}=\underbrace{X \times X \times \ldots \times X}_{n \text {-times }}$.

Let $(X, \leq)$ be a partially order set, we endow the product space $X^{3}$ with the partially orde $\leq$ defined by:

$$
\begin{array}{ll}
\text { For } & (x, y, z),(u, v, w) \in X^{3} \\
& (x, y, z) \leq(u, v, w) \Leftrightarrow x \leq u, y \geq v, z \leq w .
\end{array}
$$




\section{Main Results}

Theorem 2.1. Let $(X, d, \leq)$ be a partially ordered complete metric space, $\xi:[0, \infty) \rightarrow[0, \infty)$ is an altering distance function $\beta \in(0,1)$. Let $F: X^{3} \rightarrow X$ be a mapping such that the following conditions are satisfied:

(i) there exists $\left(x_{0}, y_{0}, z_{0}\right) \in X^{3}$ such that

$$
\left(x_{0}, y_{0}, z_{0}\right) \leq\left(F\left(x_{0}, y_{0}, z_{0}\right), F\left(y_{0}, x_{0}, y_{0}\right), F\left(z_{0}, y_{0}, x_{0}\right)\right),
$$

(ii) for $\left(x_{1}, y_{1}, z_{1}\right),\left(x_{2}, y_{2}, z_{2}\right) \in X^{3}$,

$$
x_{1} \leq x_{2}, y_{1} \geq y_{2}, z_{1} \leq z_{2}
$$

$\Rightarrow \quad F\left(x_{1}, y_{1}, z_{1}\right) \leq F\left(x_{2}, y_{2}, z_{2}\right), F\left(z_{1}, y_{1}, x_{1}\right) \leq F\left(z_{2}, y_{2}, x_{2}\right)$,

$$
F\left(y_{1}, x_{1}, y_{1}\right) \geq F\left(y_{2}, x_{2}, y_{2}\right) \text {, }
$$

(iii) If $\left(x_{n}, y_{n}, z_{n}\right) \rightarrow(x, y, z)$ is non-decreasing in first and third co-ordinates and nonincreasing in second co-ordinates then $x_{n} \leq x, y_{n} \geq y, z_{n} \leq z$ for all $n$,

(iv) $\xi\left(d\left(F\left(x_{1}, y_{1}, z_{1}\right), F\left(x_{2}, y_{2}, z_{2}\right)\right)\right) \leq \beta \xi\left(\max \left\{d\left(x_{1}, x_{2}\right)\right.\right.$,

$$
\begin{aligned}
\leq & \beta \xi\left(\operatorname { m a x } \left\{d\left(x_{1}, x_{2}\right), d\left(x_{1}, F\left(x_{1}, y_{1}, z_{1}\right)\right), d\left(x_{2}, F\left(x_{2}, y_{2}, z_{2}\right)\right),\right.\right. \\
& \left.\left.\frac{d\left(x_{2}, F\left(x_{1}, y_{1}, z_{1}\right)+d\left(x_{1}, F\left(x_{2}, y_{2}, z_{2}\right)\right)\right.}{2}\right\}\right)
\end{aligned}
$$

for all comparable $\left(x_{1}, y_{1}, z_{1}\right),\left(x_{2}, y_{2}, z_{2}\right) \in X^{3}$.

Then $F$ has a tripled fixed point.

Proof. Let $F\left(x_{0}, y_{0}, z_{0}\right)=x_{1}, F\left(y_{0}, x_{0}, y_{0}\right)=y_{1}, F\left(z_{0}, y_{0}, x_{0}\right)=z_{1}$, then by hypothesis (i), we have

$$
\begin{aligned}
& \left(x_{0}, y_{0}, z_{0}\right) \leq\left(x_{1}, y_{1}, z_{1}\right) \\
\Rightarrow \quad & x_{0} \leq x_{1}, y_{0} \geq y_{1} \text { and } z_{0} \leq z_{1} .
\end{aligned}
$$

Now by hypothesis (ii) and using equation (2.1) we have

$$
\begin{aligned}
& F\left(x_{0}, y_{0}, z_{0}\right) \leq F\left(x_{1}, y_{1}, z_{1}\right), F\left(z_{0}, y_{0}, x_{0}\right) \leq F\left(z_{1}, y_{1}, x_{1}\right) \text {. and } \\
& F\left(y_{0}, x_{0}, y_{0}\right) \geq F\left(y_{1}, x_{1}, y_{1}\right)
\end{aligned}
$$

Let $F\left(x_{1}, y_{1}, z_{1}\right)=x_{2}, F\left(y_{1}, x_{1}, y_{1}\right)=y_{2}, F\left(z_{1}, y_{1}, x_{1}\right)=z_{2}$.

Then we have

$$
x_{1} \leq x_{2}, y_{1} \geq y_{2} \text { and } z_{1} \leq z_{2} \text {. }
$$

Again by using hypothesis (ii) and equation (2.2), we have

$F\left(x_{1}, y_{1}, z_{1}\right) \leq F\left(x_{2}, y_{2}, z_{2}\right), F\left(z_{1}, y_{1}, x_{1}\right) \leq F\left(z_{2}, y_{2}, x_{2}\right)$. and

$F\left(y_{1}, x_{1}, y_{1}\right) \geq F\left(y_{2}, x_{2}, y_{2}\right)$

Continuing like this we can construct monotone non-decreasing sequences $\left\{x_{n}\right\},\left\{z_{n}\right\}$ and monotone non-increasing sequence $\left\{y_{n}\right\}$ in $X$.

That is

$$
\begin{aligned}
& x_{1} \leq x_{2} \leq x_{3} \leq \ldots \leq x_{n-1} \leq x_{n} \leq \ldots, \\
& y_{1} \geq y_{2} \geq y_{3} \geq \ldots \geq y_{n-1} \geq y_{n} \geq \ldots, \\
& z_{1} \leq z_{2} \leq z_{3} \leq \ldots \leq z_{n-1} \leq z_{n} \leq \ldots
\end{aligned}
$$

such that

$F\left(x_{n}, y_{n}, z_{n}\right)=x_{n+1}, F\left(y_{n}, x_{n}, y_{n}\right)=y_{n+1}, F\left(z_{n}, y_{n}, x_{n}\right)=z_{n+1}$ for all $n \geq 0$. 
If there exist an integer $\geq 0$ such that $x_{l}=x_{l+1}, y_{l}=y_{l+1}$ and $z_{l}=z_{l}+1$ then $\left(x_{l}, y_{l}, z_{l}\right)$ will be the coupled fixed point of $F$.

Hence we assume that either $x_{n} \neq x_{n+1}$ or $y_{n} \neq y_{n+1}$ or $z_{n} \neq z_{n+1}$ for all $n \geq 0$.

First we assume that $x_{n} \neq x_{n+1}$ for all $n \geq 0$.

Now since $x_{n} \leq x_{n+1}, y_{n} \geq y_{n+1}, z_{n} \leq z_{n+1}$, using hypothesis (iv), we have

$$
\begin{gathered}
\xi\left(d\left(x_{n+1}, x_{n+2}\right)\right)=\xi\left(d\left(F\left(x_{n}, y_{n}, z_{n}\right), F\left(x_{n+1}, y_{n+1}, z_{n+1}\right)\right)\right) \\
\leq \beta \xi\left(\operatorname { m a x } \left\{d\left(x_{n}, x_{n+1}\right), d\left(x_{n}, F\left(x_{n}, y_{n}, z_{n}\right)\right), d\left(x_{n+1}, F\left(x_{n+1}, y_{n+1}, z_{n+1}\right)\right),\right.\right. \\
\left.\left.\frac{d\left(x_{n+1}, F\left(x_{n}, y_{n}, z_{n}\right)\right)+d\left(x_{n}, F\left(x_{n+1}, y_{n+1}, z_{n+1}\right)\right.}{2}\right\}\right) \\
=\beta \xi\left(\max \left\{d\left(x_{n}, x_{n+1}\right), d\left(x_{n}, x_{n+1}\right), d\left(x_{n+1}, x_{n+2}\right), \frac{d\left(x_{n+1}, x_{n+1}\right)+d\left(x_{n}, x_{n+2}\right)}{2}\right\}\right) \\
\Rightarrow \quad \xi\left(d\left(x_{n+1}, x_{n+2}\right)\right) \leq \beta \xi\left(\max \left\{d\left(x_{n}, x_{n+1}\right), d\left(x_{n+1}, x_{n+2}\right)\right\}\right) .
\end{gathered}
$$

If we assume $d\left(x_{n}, x_{n+1}\right) \leq d\left(x_{n+1}, x_{n+2}\right)$ for some $n$, then by (2.3), we have

$$
\xi\left(d\left(x_{n+1}, x_{n+2}\right)\right) \leq \beta \xi\left(d\left(x_{n+1}, x_{n+2}\right)\right)
$$

$\Rightarrow \quad d\left(x_{n+1}, x_{n+2}\right)=0$

$\Rightarrow \quad x_{n+1}=x_{n+2}$

which is a contradiction to our assumption that $x_{n} \neq x_{n+1}$ for all $n \in N$.

So $d\left(x_{n+1}, x_{n+2}\right)<d\left(x_{n}, x_{n+1}\right)$ for all $n \geq 0$ and $\left\{d\left(x_{n}, x_{n+1}\right)\right\}$ is a monotone decreasing sequence of non-negative real numbers.

$\Rightarrow \quad \exists$ a real number $p \geq 0$ such that $d\left(x_{n}, x_{n+1}\right) \rightarrow p$ as $n \rightarrow \infty$.

Taking the limit $n \rightarrow \infty$ and using continuity of $\psi$, we have

$$
\psi(p) \leq \beta \psi(p) .
$$

$\Rightarrow \quad p=0$.

Hence

$$
\lim _{n \rightarrow \infty} d\left(x_{n}, x_{n+1}\right)=0 .
$$

Similarly if we assume $y_{n} \neq y_{n+1}$ and $z_{n} \neq z_{n+1}$, we arrive at contradictions and get

$$
\lim _{n \rightarrow \infty} d\left(y_{n}, y_{n+1}\right)=0 \text {. }
$$

and

$$
\lim _{n \rightarrow \infty} d\left(z_{n}, z_{n+1}\right)=0 \text {. }
$$

in the respective cases.

Now we will show that $\left\{x_{n}\right\}$ is a Cauchy sequence in $X$. If possible, let $\left\{x_{n}\right\}$ is not a Cauchy sequence in $X$.Then there exists an $\varepsilon>0$ such that

$$
d\left(x_{p(t)}, x_{q(t)}\right) \geq \varepsilon \text { for all } t \in N, p(t)>q(t)>t .
$$

If $p(t)$ is the smallest such natural number then we have

$$
d\left(x_{q(t)}, x_{p(t)}\right) \geq \varepsilon
$$


and

Then

$$
d\left(x_{p(t)-1}, x_{q(t)}\right)<\varepsilon .
$$

$$
\begin{aligned}
& d\left(x_{q(t)}, x_{p(t)}\right) \leq d\left(x_{q(t)}, x_{p(t)-1}\right)+d\left(x_{p(t)-1}, x_{p(t)}\right) \\
& <\varepsilon+d\left(x_{p(t)-1}, x_{p(t)}\right) \quad(\operatorname{using}(2.9)) \\
\Rightarrow \quad & d\left(x_{q(t)}, x_{p(t)}\right)<\varepsilon+d\left(x_{p(t)-1}, x_{p(t)}\right) .
\end{aligned}
$$

Taking limit as $t \rightarrow \infty$ and using (2.5), equations (2.8) and (2.10) jointly give

$$
\lim _{t \rightarrow \infty} d\left(x_{q(t)}, x_{p(t)}\right)=\varepsilon .
$$

Now

$$
\begin{aligned}
& d\left(x_{q(t)}, x_{p(t)}\right) \leq d\left(x_{q(t)}, x_{q(t)+1}\right)+d\left(x_{q(t)+1}, x_{p(t)+1}\right)+d\left(x_{p(t)+1}, x_{p(t)}\right) \\
\Rightarrow \quad & \lim _{t \rightarrow \infty} d\left(x_{q(t)+1}, x_{p(t)+1}\right) \geq \varepsilon .
\end{aligned}
$$

Also

$$
d\left(x_{q(t)+1}, x_{p(t)+1}\right) \leq d\left(x_{q(t)+1}, x_{q(t)}\right)+d\left(x_{q(t)}, x_{p(t)}\right)+d\left(x_{p(t)}, x_{p(t)+1}\right) .
$$

Again taking limit $t \rightarrow \infty$ and using equations (2.5) and (2.11), we get

$$
\lim _{t \rightarrow \infty} d\left(x_{q(t)+1}, x_{p(t)+1}\right) \leq \varepsilon .
$$

Combining (2.12) and (2.13), we have

$$
\lim _{t \rightarrow \infty} d\left(x_{n(t)+1}, x_{p(t)+1}\right)=\varepsilon \text {. }
$$

Again

and

$$
d\left(x_{q(t)}, x_{p(t)}\right) \leq d\left(x_{q(t)}, x_{p(t)+1}\right)+d\left(x_{p(t)+1}, x_{p(t)}\right)
$$

$$
d\left(x_{q(t)}, x_{p(t)+1}\right) \leq d\left(x_{q(t)}, x_{p(t)}\right)+d\left(x_{p(t)}, x_{p(t)+1}\right) .
$$

Taking limit as $t \rightarrow \infty$ and using (2.5) and (2.11), we get

$\lim _{t \rightarrow \infty} d\left(x_{q(t)}, x_{p(t)+1}\right)=\varepsilon$.

Again

and

$$
d\left(x_{p(t)}, x_{q(t)}\right) \leq d\left(x_{p(t)}, x_{q(t)+1}\right)+d\left(x_{q(t)+1}, x_{q(t)}\right)
$$

$$
d\left(x_{p(t)}, x_{q(t)+1}\right) \leq d\left(x_{p(t)}, x_{q(t)}\right)+d\left(x_{q(t)}, x_{q(t)+1}\right) .
$$

Taking limit as $t \rightarrow \infty$ and using (2.5) and (2.11), we get

$$
\lim _{t \rightarrow \infty} d\left(x_{p(t)}, x_{q(t)+1}\right)=\varepsilon .
$$

Now $p(t)>q(t) \Rightarrow x_{p(t)} \geq x_{q(t)}, y_{p(t)} \leq y_{q(t)}, z_{p(t)} \geq z_{q(t)}$ for all $t \in N$.

So using hypothesis (iv), we have

$$
\begin{aligned}
& \xi\left(d\left(x_{q(t)+1}, x_{p(t)+1}\right)\right)=\xi\left(d\left(F\left(x_{q(t)}, y_{q(t)}, z_{q(t)}\right), F\left(x_{p(t)}, y_{p(t)}, z_{p(t)}\right)\right)\right. \\
& \leq \beta \xi\left(\operatorname { m a x } \left\{d\left(x_{q(t)}, x_{p(t)}\right), d\left(x_{q(t)}, F\left(x_{q(t)}, y_{q(t)}, z_{q(t)}\right)\right), d\left(x_{p(t)}, F\left(x_{p(t)}, y_{p(t)}, z_{p(t)}\right)\right),\right.\right. \\
& \left.\left.\quad \frac{d\left(x_{p(t)}, F\left(x_{q(t)}, y_{q(t)}, z_{q(t)}\right)\right)+d\left(x_{q(t)}, F\left(x_{p(t)}, y_{p(t)}, z_{p(t)}\right)\right.}{2}\right\}\right) \\
& =\beta \xi\left(\max \left\{d\left(x_{q(t)}, x_{p(t)}\right), d\left(x_{q(t)}, x_{q(t)+1}\right), d\left(x_{p(t)}, x_{p(t)+1}\right), \frac{d\left(x_{p(t)}, x_{q(t)+1}\right)+d\left(x_{q(t)}, x_{p(t)+1}\right)}{2}\right\}\right) . \text { Taking }
\end{aligned}
$$

limit as $t \rightarrow \infty$ and using (2.5), (2.11) and (2.14)-(2.16), we get $\xi(\varepsilon) \leq \beta \xi(\varepsilon)$, which is not possible by definition of $\xi$ and choice of $\beta$. Hence $\left\{x_{n}\right\}$ is a Cauchy sequence in $X$. Similarly 
$\left\{y_{n}\right\}$ and $\left\{z_{n}\right\}$ are also Cauchy sequence in $X$. But $X$ is given to be complete. So there exist such that

$$
x, y, z \in X x_{n} \rightarrow x, y_{n} \rightarrow y, z_{n} \rightarrow z \text { as } n \rightarrow \infty .
$$

Then by hypothesis (iii) $x_{n} \leq x, y_{n} \geq y$ and $z_{n} \leq z$ for all $n$.

So using hypothesis (iv), we get

$$
\begin{aligned}
& \xi\left(d\left(x_{n+1}, F(x, y, x)\right)\right)=\xi\left(d\left(F\left(x_{n}, y_{n}, z_{n}\right), F(x, y, z)\right)\right) \\
& \leq \beta \xi\left(\operatorname { m a x } \left\{d\left(x_{n}, x\right), d\left(x_{n}, F\left(x_{n}, y_{n}, z_{n}\right)\right), d(x, F(x, y, z)),\right.\right. \\
&\left.\left.\frac{d\left(x, F\left(x_{n}, y_{n}, z_{n}\right)\right)+d\left(x_{n}, F(x, y, z)\right.}{2}\right\}\right) \\
&=\beta \xi\left(\max \left\{d\left(x_{n}, x\right), d\left(x_{n}, x_{n+1}\right), d(x, F(x, y, z)), \frac{d\left(x, x_{n+1}\right)+d\left(x_{n}, F(x, y, z)\right)}{2}\right\}\right) . \text { Now taking limit as } \\
& n \rightarrow \infty \text { and using }(2.5) \text { and }(2.17), \text { we get } \\
& \quad \xi(d(x, F(x, y, z))) \leq \beta \xi(d(x, F(x, y, z))) \\
& \Rightarrow \quad d(x, F(x, y, z))=0 \\
& \Rightarrow \quad F(x, y, z)=x .
\end{aligned}
$$

Since $y_{n} \geq y$ and $x_{n} \leq x$, so again using hypothesis (iv), we have

$\xi\left(d\left(y_{n+1}, F(y, x, y)\right)\right)=\xi\left(d\left(F\left(y_{n}, x_{n}, y_{n}\right), F(y, x, y)\right)\right)$

$\leq \beta \xi\left(\max \left\{d\left(y_{n}, y\right), d\left(y_{n}, F\left(y_{n}, x_{n}, y_{n}\right)\right), d(x, F(y, x, y))\right.\right.$,

$$
\begin{aligned}
& \left.\left.\qquad \frac{d\left(z, F\left(z_{n}, y_{n}, x_{n}\right)\right)+d\left(z_{n}, F(z, y, x)\right.}{2}\right\}\right) \\
& =\beta \xi\left(\max \left\{d\left(z_{n}, z\right), d\left(z_{n}, z_{n+1}\right), d(z, F(z, y, x)), \frac{d\left(z, z_{n+1}\right)+d\left(z_{n}, F(z, y, x)\right)}{2}\right\}\right) . \\
& n \rightarrow \infty \text { and using }(2.6) \text { and }(2.17), \text { we get } \\
& \xi(d(y, F(y, x, y))) \leq \beta \xi(d(y, F(y, x, y))) \\
& \Rightarrow \quad d(y, F(y, x, y))=0 \\
& \Rightarrow \quad F(y, x, y)=y .
\end{aligned}
$$

Finally, since $z_{n} \leq z, y_{n} \geq y$ and $x_{n} \leq x$ for all $n$, so again using hypothesis (iv), we get

$$
\begin{aligned}
& \xi\left(d\left(z_{n+1}, F(z, y, x)\right)\right)=\xi\left(d\left(F\left(z_{n}, y_{n}, x_{n}\right), F(z, y, x)\right)\right) \\
& \leq \beta \xi\left(\operatorname { m a x } \left\{d\left(z_{n}, z\right), d\left(z_{n}, F\left(z_{n}, y_{n}, x_{n}\right)\right), d(z, F(z, y, x)),\right.\right. \\
& \left.\left.\frac{d\left(z, F\left(z_{n}, y_{n}, x_{n}\right)\right)+d\left(z_{n}, F(z, y, x)\right.}{2}\right\}\right) \\
& =\beta \xi\left(\max \left\{d\left(z_{n}, z\right), d\left(z_{n}, z_{n+1}\right), d(z, F(z, y, x)), \frac{d\left(z, z_{n+1}\right)+d\left(z_{n}, F(z, y, x)\right)}{2}\right\}\right) .
\end{aligned}
$$

Now taking limit as $n \rightarrow \infty$ and using (2.7) and (2.17), we get

$$
\begin{aligned}
& \xi(d(z, F(z, y, x))) \leq \beta \xi(d(z, F(z, y, x))) \\
\Rightarrow \quad & d(z, F(z, y, x))=0
\end{aligned}
$$$$
\Rightarrow \quad F(z, y, x)=z \text {. }
$$

Thus from (2.18)-(2.20), we conclude that there exist $(x, y, z) \in X^{3}$ such that $F(x, y, z)=x, F(y, x, y)=y$ and $F(z, y, x)=z$ which implies that $(x, y, z)$ is a tripled fixed point of $F$.

In the following theorem we will establish the existence of tripled fixed point for a continuous mapping after the removal of hypothesis (iii) from Theorem 2.1. 
Theorem 2.2. Let $(X, d, \leq)$ be a partially ordered complete metric space, $\xi:[0, \infty) \rightarrow[0, \infty)$ is an altering distance function and $\beta \in(0,1)$. Let $F: X^{3} \rightarrow X$ be a continuous mapping such that the following conditions are satisfied:

(i) there exist $\left(x_{0}, y_{0}, z_{0}\right) \in X^{3}$ such that

$\left(x_{0}, y_{0}, z_{0}\right) \leq\left(F\left(x_{0}, y_{0}, z_{0}\right), F\left(y_{0}, x_{0}, y_{0}\right), F\left(z_{0}, y_{0}, x_{0}\right)\right)$,

(ii) for $\left(x_{1}, y_{1}, z_{1}\right),\left(x_{2}, y_{2}, z_{2}\right) \in X^{3}$,

$$
\begin{aligned}
& \left(x_{1}, y_{1}, z_{1}\right) \leq\left(x_{2}, y_{2}, z_{2}\right) \Rightarrow F\left(x_{1}, y_{1}, z_{1}\right) \leq F\left(x_{2}, y_{2}, z_{2}\right), \\
& F\left(y_{1}, x_{1}, y_{1}\right) \geq F\left(y_{2}, x_{2}, y_{2}\right) \text { and } F\left(z_{1}, y_{1}, x_{1}\right) \leq F\left(z_{2}, y_{2}, x_{2}\right),
\end{aligned}
$$

(iii) $\xi\left(d\left(F\left(x_{1}, y_{1}, z_{1}\right), F\left(x_{2}, y_{2}, z_{2}\right)\right) \leq \beta \xi\left(\max \left\{d\left(x_{1}, x_{2}\right)\right.\right.\right.$,

$$
\begin{gathered}
d\left(x_{1}, F\left(x_{1}, y_{1}, z_{1}\right), d\left(x_{2}, F\left(x_{2}, y_{2}, z_{2}\right)\right),\right. \\
\left.\left.\frac{d\left(x_{2}, F\left(x_{1}, y_{1}, z_{1}\right)+d\left(x_{1}, F\left(x_{2}, y_{2}, z_{2}\right)\right)\right.}{2}\right\}\right)
\end{gathered}
$$

for all comparable $\left(x_{1}, y_{1}, z_{1}\right),\left(x_{2}, y_{2}, z_{2}\right) \in X^{3}$

Then $F$ has a tripled fixed point.

Proof. From the proof of Theorem 2.1, we find sequences $\left\{x_{n}\right\},\left\{y_{n}\right\}$ and $\left\{z_{n}\right\}$ such that

$$
\lim _{n \rightarrow \infty} x_{n}=x, \lim _{n \rightarrow \infty} y_{n}=y \text { and } \lim _{n \rightarrow \infty} z_{n}=z \text {. }
$$

Then continuity of $F$ implies that

$$
\begin{aligned}
& x=\lim _{n \rightarrow \infty} x_{n+1}=\lim _{n \rightarrow \infty}\left(x_{n}, y_{n}, z_{n}\right)=F(x, y, z), \\
& y=\lim _{n \rightarrow \infty} y_{n+1}=\lim _{n \rightarrow \infty}\left(y_{n}, x_{n}, y_{n}\right)=F(y, x, y)
\end{aligned}
$$

and

$$
z=\lim _{n \rightarrow \infty} z_{n+1}=\lim _{n \rightarrow \infty}\left(z_{n}, y_{n}, x_{n}\right)=F(z, y, x)
$$

Thus we find $(x, y, z) \in X^{3}$ such that

$F(x, y, z)=x, F(y, x, y)=y$ and $F(z, y, x)=z$

$\Rightarrow \quad(x, y, z)$ is a tripled fixed point of $F$.

Theorem 2.3. Let $(X, d, \leq)$ be a partially ordered complete metric space and $\xi:[0, \infty) \rightarrow[0, \infty)$ is an altering distance function. Let: $F: X^{3} \rightarrow X$ be a mapping such that the following conditions are satisfied:

(i) there exist $\left(x_{0}, y_{0}, z_{0}\right) \in X^{3}$ such that

$$
\left(x_{0}, y_{0}, z_{0}\right) \leq\left(F\left(x_{0}, y_{0}, z_{0}\right), F\left(y_{0}, x_{0}, y_{0}\right), F\left(z_{0}, y_{0}, x_{0}\right)\right) \text {, }
$$

(ii) for $\left(x_{1}, y_{1}, z_{1}\right),\left(x_{2}, y_{2}, z_{2}\right) \in X^{2}$,

$$
\left(x_{1}, y_{1}, z_{1}\right) \leq\left(x_{2}, y_{2}, z_{2}\right) \Rightarrow F\left(x_{1}, y_{1}, z_{1}\right) \leq F\left(x_{2}, y_{2}, z_{2}\right) \text {, }
$$

$F\left(y_{1}, x_{1}, y_{1}\right) \geq F\left(y_{2}, x_{2}, y_{2}\right), F\left(z_{1}, y_{1}, x_{1}\right) \leq F\left(z_{2}, y_{2}, x_{2}\right)$,

(iii) If $\left(x_{n}, y_{n}, z_{n}\right) \rightarrow(x, y, z)$ is monotone non-decreasing in first and third coordinates and nonincreasing in second co-ordinate, then

$$
x_{n} \leq x, y_{n} \geq y \text { and } z_{n} \leq z \text { for all } n \text {. }
$$

(iv) $\xi\left(d\left(F\left(x_{1}, y_{1}, z_{1}\right), F\left(x_{2}, y_{2}, z_{2}\right)\right) \leq \beta \xi\left(\max \left\{d\left(x_{1}, x_{2}\right)\right.\right.\right.$, 


$$
\begin{gathered}
d\left(x_{1}, F\left(x_{1}, y_{1}, z_{1}\right)\right), d\left(x_{2}, F\left(x_{2}, y_{2}, z_{2}\right)\right), \\
\left.\left.\frac{d\left(x_{2}, F\left(x_{1}, y_{1}, z_{1}\right)\right)+d\left(x_{1}, F\left(x_{2}, y_{2}, z_{2}\right)\right)}{2}\right\}\right) \\
-\eta\left(\max \left\{d\left(x_{1}, x_{2}\right), d\left(x_{2}, F\left(x_{2}, y_{2}, z_{2}\right)\right)\right\}\right)
\end{gathered}
$$

for all comparable $\left(x_{1}, y_{1}, z_{1}\right),\left(x_{2}, y_{2}, z_{2}\right) \in X^{3}$,

where $\eta:[0, \infty) \rightarrow[0, \infty)$ is any continuous function with $\eta(t)=0$ iff $t=0$. Then $F$ has a tripled fixed point.

Proof. We will construct the same sequences $\left\{x_{n}\right\},\left\{y_{n}\right\}$ and $\left\{z_{n}\right\}$ as in Theorem 2.1.

Now if there exist a positive integer $l$ such that $x_{l}=x_{l+1}, y_{l}=y_{l_{+1}}$ and $z_{l}=z_{l+1}$ then $\left(x_{l}, y_{l}, z_{l}\right)$ will be a tripled fixed point of $F$.

Hence we assume that either $x_{n} \neq x_{n+1}$ or $y_{n} \neq y_{n+1}$ or $z_{n} \neq z_{n+1}$ for all $n$.

First we assume that $x_{n} \neq x_{n+1}$ for all $n$.

Now since $x_{n} \leq x_{n+1}, y_{n} \geq y_{n+1}$ and $z_{n} \leq z_{n+1}$, so using hypothesis (iv), we have

$$
\begin{aligned}
\xi\left(d\left(x_{n+1}, x_{n+2}\right)\right)=\xi & \left(d\left(F\left(x_{n}, y_{n}, z_{n}\right), F\left(x_{n+1}, y_{n+1}, z_{n+1}\right)\right)\right) \\
\leq \xi(\max \{d & \left(x_{n}, x_{n+1}\right), d\left(x_{n}, F\left(x_{n}, y_{n}, z_{n}\right)\right), d\left(x_{n+1}, F\left(x_{n+1}, y_{n+1}, z_{n+1}\right)\right), \\
& \left.\left.\left.\frac{d\left(x_{n+1}, F\left(x_{n}, y_{n}, z_{n}\right)\right)+d\left(x_{n}, F\left(x_{n+1}, y_{n+1}, z_{n+1}\right)\right.}{2}\right\}\right)\right) \\
- & \eta\left(\max \left\{d\left(x_{n}, x_{n+1}\right), d\left(x_{n+1}, F\left(x_{n+1}, y_{n+1}, z_{n+1}\right)\right)\right\}\right)
\end{aligned}
$$

$$
=\xi\left(\max \left\{d\left(x_{n}, x_{n+1}\right), d\left(x_{n}, x_{n+1}\right), d\left(x_{n+1}, x_{n+2}\right), \frac{d\left(x_{n+1}, x_{n+1}\right)+d\left(x_{n}, x_{n+2}\right)}{2}\right\}\right)
$$

$\left.-\eta\left(\max \left\{d\left(x_{n}, x_{n+1}\right), d\left(x_{n+1}, x_{n+2}\right)\right)\right\}\right)$

$$
\begin{gathered}
\quad \leq \xi\left(\max \left\{d\left(x_{n}, x_{n+1}\right), d\left(x_{n+1}, x_{n+2}\right)\right\}\right)-\eta\left(\max \left\{d\left(x_{n}, x_{n+1}\right), d\left(x_{n+1}, x_{n+2}\right)\right\}\right) \\
\Rightarrow \quad-\eta\left(d\left(x_{n+1}, x_{n+2}\right)\right) \leq \xi\left(\max \left\{d\left(x_{n}, x_{n+1}\right), d\left(x_{n+1}, x_{n+2}\right)\right\}\right) \\
\left.-\eta\left(\max \left\{d\left(x_{n}, x_{n+1}\right), d\left(x_{n+1}, x_{n+2}\right)\right)\right\}\right) .
\end{gathered}
$$

Suppose $d\left(x_{n}, x_{n+1}\right) \leq d\left(x_{n+1}, x_{n+2}\right)$ for some $n$.

Then (2.21) implies

$$
\begin{array}{ll} 
& \xi\left(d\left(x_{n+1}, x_{n+2}\right)\right) \leq \xi\left(d\left(x_{n+1}, x_{n+2}\right)\right)-\eta\left(d\left(x_{n+1}, x_{n+2}\right)\right) \\
\Rightarrow & \eta\left(d\left(x_{n+1}, x_{n+2}\right)\right) \leq 0 \\
\Rightarrow & d\left(x_{n+1}, x_{n+2}\right)=0 \\
\Rightarrow & x_{n+1}=x_{n+2} \text { which is contradiction to our assumption }
\end{array}
$$
that $\Rightarrow x_{n} \neq x_{n+1}$ for all $n$.

$d\left(x_{n+1}, x_{n+2}\right)<\left(x_{n}, x_{n+1}\right)$ for all $n$

and $\left\{d\left(x_{n}, x_{n+1}\right)\right\}$ is a monotone decreasing sequence of non-negative real numbers

$\Rightarrow \quad$ there exist a $p \geq 0$ such that

$$
\lim _{n \rightarrow \infty} d\left(x_{n}, x_{n+1}\right)=p .
$$

Now using (2.21) and (2.22) we have

$$
\xi\left(d\left(x_{n+1}, x_{n+2}\right)\right) \leq \xi\left(d\left(x_{n}, x_{n+1}\right)\right)-\eta\left(d\left(x_{n}, x_{n+1}\right)\right) .
$$

Taking limit as $n \rightarrow \infty$ and using (2.23), we get

$\xi(p) \leq \xi(p)-\eta(p)$ (using continuity of $\xi$ and $\eta$ )

which will give a contradiction unless $p=0$. 
So

$$
\lim _{n \rightarrow \infty} d\left(x_{n}, x_{n+1}\right)=0 \text {. }
$$

Similarly if we assume $y_{n} \neq y_{n+1}$ and $z_{n} \neq z_{n+1}$, then we will arrive at contradiction and get

$$
\lim _{n \rightarrow \infty} d\left(y_{n}, y_{n+1}\right)=0
$$

and

$$
\lim _{n \rightarrow \infty} d\left(z_{n}, z_{n+1}\right)=0
$$

in the respective cases.

Now we will show that $\left\{x_{n}\right\}$ is a Cauchy sequence in $X$. If we assume that $\left\{x_{n}\right\}$ is not a Cauchy sequence in $X$ then following the arguments of Theorem 2.1, we have two sequences of positive integers $\{p(t)\}$ and $\{q(t)\}$ such that $p(t)>q(t)>t$ for all $t$

$$
\begin{aligned}
& \lim _{t \rightarrow \infty} d\left(x_{p(t)}, x_{q(t)}\right)=\varepsilon, \\
& \lim _{t \rightarrow \infty} d\left(x_{p(t)+1}, x_{q(t)+1}\right)=\varepsilon, \\
& \lim _{t \rightarrow \infty} d\left(x_{p(t)}, x_{q(t)+1}\right)=\varepsilon, \\
& \lim _{t \rightarrow \infty} d\left(x_{q(t)}, x_{p(t)+1}\right)=\varepsilon .
\end{aligned}
$$

Now $p(t)>q(t) \Rightarrow x_{p(t)} \geq x_{q(t)}, y_{p(t)} \leq y_{q(t)}, z_{p(t)} \geq z_{q(t)}$ for all $t \in N$.

So using hypothesis (vi), we get

$$
\begin{aligned}
& \xi\left(d\left(x_{q(t)+1}, x_{p(t)+1}\right)\right)=\xi\left(d\left(F\left(x_{q(t)}, y_{q(t)}, z_{q(t)}\right), F\left(x_{p(t)}, y_{p(t)}, z_{p(t)}\right)\right)\right) \\
& \leq \xi\left(\operatorname { m a x } \left\{d\left(x_{q(t)}, x_{p(t)}\right), d\left(x_{q(t)}, F\left(x_{q(t)}, y_{q(t)}, z_{q(t)}\right)\right), d\left(x_{p(t)}, F\left(x_{p(t)}, y_{p(t)}, z_{p(t)}\right)\right),\right.\right. \\
& \quad-\eta\left(\max \left\{d\left(x_{q(t)}, x_{p(t)}\right), d\left(x_{p(t)}, F\left(x_{p(t)}, y_{p(t)}, z_{p(t)}\right)\right)\right\}\right) \\
& =\xi\left(\max \left\{d\left(x_{q(t)}, x_{p(t)}\right), d\left(x_{q(t)}, x_{q(t)+1}\right), d\left(x_{p(t)}, x_{p(t)+1}\right), \frac{d\left(x_{p(t)}, x_{q(t)+1}\right)+d\left(x_{q(t)}, x_{p(t)+1}\right)}{2}\right\}\right) \\
& \left.-\eta\left(\max \left\{d\left(x_{q(t)}, x_{p(t)}\right), d\left(x_{p(t)}, x_{p(t)+1}\right)\right)\right\}\right) .
\end{aligned}
$$

Taking limit as $t \rightarrow \infty$ and using (2.24) and (2.27)-(2.30), we have $\xi(\varepsilon) \leq \xi(\varepsilon)-\eta(\varepsilon)$, which is not possible by definition of $\eta$.

Hence $\left\{x_{n}\right\}$ is a Cauchy sequence in $X$.

Similarly $\left\{y_{n}\right\}$ and $\left\{z_{n}\right\}$ are also Cauchy sequences in $X$.

But $X$ is complete so there exists $x, y, z \in X$ such that

$$
x_{n} \rightarrow x, y_{n} \rightarrow y \text { and } z_{n} \rightarrow z \text { as } n \rightarrow \infty \text {. }
$$

Then by hypothesis (iii) $x_{n} \leq x, y_{n} \geq y$ and $z_{n} \leq z$ for all $n$.

So we can use condition (iv) and get

$$
\begin{gathered}
\xi\left(d\left(x_{n+1}, F(x, y, z)\right)\right)=\xi\left(d\left(F\left(x_{n}, y_{n}, z_{n}\right), F(x, y, z)\right)\right) \\
\leq \xi\left(\operatorname { m a x } \left\{d\left(x_{n}, x\right), d\left(x_{n}, F\left(x_{n}, y_{n}, z_{n}\right)\right), d(x, F(x, y, z)),\right.\right. \\
\left.\left.\frac{d\left(x_{n}, F(x, y, z)\right)+d\left(x, F\left(x_{n}, y_{n}, z_{n}\right)\right)}{2}\right\}\right) \\
-\eta\left(\max \left\{d\left(x_{n}, x\right), d\left(x_{n}, F(x, y, z)\right)\right\}\right) .
\end{gathered}
$$

Taking limit as $n \rightarrow \infty$ and using equations (2.24) and (2.31), we get

$$
\xi(d(x, F(x, y, z))) \leq \xi(d(x, F(x, y, z)))-\eta(d(x, F(x, y, z)))
$$


which leads to a contradiction unless $d(x, F(x, y, z))=0$.

$$
\Rightarrow \quad F(x, y, z)=x \text {. }
$$

Similarly

$$
F(y, x, y)=y \text { and } F(z, y, x)=z \text {. }
$$

Combining equations (2.32) and (2.33) we get $(x, y, z)$ is tripled fixed point of $F$.

In the following theorem we will show existence of tripled fixed point for a continuous mapping after the removal of hypothesis (iii) of Theorem 2.3.

Theorem 2.4. Let $(X, d, \leq)$ be a partially ordered complete metric space and $\xi:[0, \infty) \rightarrow[0, \infty)$ be an altering distance function. $F: X^{3} \rightarrow X$ be a continuous mapping such that the following. Let conditions are satisfied:

(i) there exist $\left(x_{0}, y_{0}, z_{0}\right) \in X^{3}$ such that

$$
\left(x_{0}, y_{0}, z_{0}\right) \leq\left(F\left(x_{0}, y_{0}, z_{0}\right), F\left(y_{0}, x_{0}, y_{0}\right), F\left(z_{0}, y_{0}, x_{0}\right)\right) \text {, }
$$

(ii) for $\left(x_{1}, y_{1}, z_{1}\right),\left(x_{2}, y_{2}, z_{2}\right) \in X^{2}$,

$$
\begin{aligned}
& \left(x_{1}, y_{1}, z_{1}\right) \leq\left(x_{2}, y_{2}, z_{2}\right) \Rightarrow F\left(x_{1}, y_{1}, z_{1}\right) \leq F\left(x_{2}, y_{2}, z_{2}\right), \\
& F\left(y_{1}, x_{1}, y_{1}\right) \geq F\left(y_{2}, x_{2}, y_{2}\right) \text { and } F\left(z_{1}, y_{1}, x_{1}\right) \leq F\left(z_{2}, y_{2}, x_{2}\right),
\end{aligned}
$$

$$
\begin{aligned}
& \xi\left(d \left(F\left(x_{1}, y_{1}, z_{1}\right), F(\right.\right.\left.\left.x_{2}, y_{2}, z_{2}\right)\right) \leq \beta \xi\left(\operatorname { m a x } \left\{d\left(x_{1}, x_{2}\right),\right.\right. \\
& d\left(x_{1}, F\left(x_{1}, y_{1}, z_{1}\right), d\left(x_{2}, F\left(x_{2}, y_{2}, z_{2}\right),\right.\right. \\
&\left.\left.\frac{d\left(x_{2}, F\left(x_{1}, y_{1}, z_{1}\right)\right)+d\left(x_{1}, F\left(x_{2}, y_{2}, z_{2}\right)\right)}{2}\right\}\right) \\
&-\eta\left(\max \left\{d\left(x_{1}, x_{2}\right), d\left(x_{2}, F\left(x_{2}, y_{2}, z_{2}\right)\right)\right\}\right)
\end{aligned}
$$

for all comparable $\left(x_{1}, y_{1}, z_{1}\right),\left(x_{2}, y_{2}, z_{2}\right) \in X^{3}$,

where $\eta:[0, \infty) \rightarrow[0, \infty)$ is any continuous function with $\eta(t)=0$ iff $t=0$. Then $F$ has a tripled fixed point.

Proof. From the proof of Theorem 2.1, we find sequences $\left\{x_{n}\right\},\left\{y_{n}\right\}$ and $\left\{z_{n}\right\}$ such that $\lim _{n \rightarrow \infty} x_{n} \rightarrow x, \lim _{n \rightarrow \infty} y_{n} \rightarrow y$ and $\lim _{n \rightarrow \infty} z_{n} \rightarrow z$.

Then continuity of $F$ implies that

$$
\begin{aligned}
& x=\lim _{n \rightarrow \infty} x_{n+1}=\lim _{n \rightarrow \infty} F\left(x_{n}, y_{n}, z_{n}\right)=F(x, y, z), \\
& y=\lim y_{n+1}=\lim F\left(y_{n}, x_{n}, y_{n}\right)=F(y, x, y),
\end{aligned}
$$

and

$$
z=\lim z_{n+1}=\lim F\left(z_{n}, y_{n}, x_{n}\right)=F(z, y, x)
$$

$$
{ }_{n \rightarrow \infty} \quad n \rightarrow \infty
$$

that is $\exists(x, y, z) \in X^{3}$ such that

$$
F(x, y, z)=x, F(y, x, y)=y \text { and } F(z, y, x)=z \text {. }
$$

$\Rightarrow \quad(x, y, z)$ is tripled fixed point of $F$. 


\section{References}

[1] H. Ayadi and M. Abbas, Tripled coincidence and fixed point results in partial metric spaces, Applied General Topology, 13 (2) (2012), 193-206.

[2] V. Berinde and M. Borcut, Tripled fixed point thoerems for contractive type mappings in partially ordered metric spaces, Nonlinear Anal., 74(15) (2011), 4889-4897.

[3] A. Cabada and J.J. Nieto, Fixed points and approximate solutions for nonlinear operators equations, J. Comput. Appl. Math., 113 (2000), 17-25.

[4] B.S. Choudhury, Certain fixed point theorems on complete metric spaces, Soochow J. Math, 22 (3) (1996), 427-434.

[5] B.S. Choudhury, A common unique fixed point result in metric spaces involving generalized altering distances, Math. Commun, 10 (2005), 105-110.

[6] B.S. Choudhury and K. Das, A coincidence point result in Menger spaces using a control functoin, Chaos Solitons Fract., 42 (2009), 3058-3063.

[7] B.S. Choudhury and P.N. Dutta, Common fixed points for fuzzy mappings using generlaized altering distances, Soochow J. Math., 31 (1) (2005), 71-81.

[8] Z. Dricia, F.A. McRacb and J. Vasundhara Devi, Fixed point theorems in partially ordered metric spaces for operators with PPF dependence, Nonlinear Anal., 67(2007), 641-647.

[9] J. Harjani and K. Sadarngani, Generalized contractions in partially ordered metric spaces and applications to ordinary differential equations, Nonlinear Anal., 72 (2010), 1188-1197.

[10] S. Hong, Fixed points of multivalued operators in ordered metric spaces with applications, Nonlinear Anal., 72 (2010), 3929-3942.

[11] Z. Kadelburg, M. Pavlovic and S. Radenovic, Common fixed point theorems for ordered contractions and quasi contractions in ordered cone metric spaces, Comput. Math. Appl., 59 (9) (2010), 3148-3159.

[12] E. Karapinar, Tripled fixed point theorems in partially ordered metric spaces, Stud. Univ. Babes-Bolyai Math., 58 (1) (2013), 75-85.

[13] M.S. Khan, M. Swaleh and S. Sessa, Fixed points theorem by altering distances between the points, Bull. Austral Math. Soc., 30 (1984), 1-9.

[14] N.V. Luong and N.X. Thuan, Coupled fixed points in partially ordered metric spaces, Bull.Math. Anal. Appl., 4 (2) (2010), 16-24.

[15] D. Mihet, Altering distances in probabilistic Menger spaces, Nonlinear Anal., 71 (2009), 2734-2738.

[16] B. Monjardet, Metrices on partially ordred set - A survey, Discrete Mathematics, 35 (1981), 173-184.

[17] S.V.R. Naidu, Some fixed point theorems in metric spaces by altering distances, Czechoslovak Math. J., 53 (1) (2003), 205-212.

[18] J.J. Nieto and R.R. Lopez, Contractive mapping theorems in partially ordered sets and applications to ordinary differential equaitons, Order, 22 (2005), 223-239.

[19] A.C.M. Ran and M.C.B. Reurings, A fixed point theorem in partially ordered sets and some application to matrix equation, Proc.Amer. Math. Soc., 132 (2004), 1435-1443.

[20] K.P.R. Rao, G.M.V. Kishore and P.R. Shobhuna Babu, Tripled coincidence point theorems for multivalued maps in partially ordered metric spaces, Universal Journal of Computaional Mathematics, 1 (2) (2013), 19-23.

[21] K.P.R. Sastry and G.V.R. Babu, Some fixed point theorems by altering distances between the points, Ind. J. Pure Appl. Math., 30 (6) (1999), 641-647.

[22] X. Zhang, Fixed point theorems for multivalued monotone mappings in ordered metric space, Appl. Math. Lett., 23 (2010), 235-240. 